\title{
Non-autonomous Second Order Differential Inclusions with a Stabilizing Effect
}

\author{
Tiziana Cardinali@ and Eleonora De Angelis
}

\begin{abstract}
In this paper we prove the existence of mild solutions for a problem governed by a semilinear non-autonomous second order differential inclusion where a stabilization of the solution is expected due to the control of the reaction term. In order to obtain our existence theorem, first we study a more general problem with a differential inclusion which involves a perturbation guided by an operator $N: I \rightarrow C(C(I ; X) ; X)$, where $X$ is a Banach space. Finally we show an illustrative example of application of our results to a problem involving a wave equation.
\end{abstract}

Mathematics Subject Classification. 34A60, 34G25.

Keywords. Semilinear non-autonomous second order differential inclusions, nonlocal conditions, fundamental system, fixed point method, stabilizing effect.

\section{Introduction}

The goal of this work is to consider theoretical mathematical models governed by differential inclusions which adapt themselves to study controllability problems in medicine, in life sciences or in other real phenomena. In particular, in this paper we prove the existence of mild solutions for the following nonlocal abstract problem for a semilinear non-autonomous second order differential inclusion

$$
(N L P)\left\{\begin{array}{l}
u^{\prime \prime}(t) \in A(t) u(t)+F\left(t, u_{R}(t)\right), t \in I \\
u(0)=g(u) \\
u^{\prime}(0)=h(u)
\end{array}\right.
$$


being

$$
u_{R}(t)=\left\{\begin{array}{l}
u(t), \quad\|u(t)\|_{X} \leq R \\
R \frac{u(t)}{\|u(t)\|_{X}}, \quad\|u(t)\|_{X}>R
\end{array}\right.
$$

where it happens that the reaction term stabilizes itself at large values over $R$ of the system states. With regard to differential equations or differential inclusions subject to general nonlocal initial conditions, we mention Byszewski's pioneering work [2]. In this topic, many results were obtained subsequently for first and second order differential equations/inclusions (see, for example, García-Falset [8], García-Falset and Reich [9] for first order equations; Aizicovici and Staicu [1], Paicu and Vrabie [18] for first order differential inclusions; Henríquez et al. [11] for second order differential equations and Cardinali and Gentili [3] for second order differential inclusions). The motivation of these studies lies in the fact that problems with nonlocal conditions represent mathematical models for the evolution of various phenomena: nonlocal pharmacokinetics, nonlocal neural networks, nonlocal pollution, nonlocal combustion (see McKibben [16], Sect. 10.2).

Comparing this work to above-mentioned papers, the novelty is that the differential semilinear inclusion governing (NLP) problem presents a nonlinear perturbation term which has an effect of stabilization on the solution. In our opinion these studies can be important in order to investigate, for example, the dynamics of a desease under drug treatment or the bacterial persistance under the effect of an antibiotic therapy or to prove that a vaccine is a good candidate for immunotherapy. Let us recall that in medicine an impressive body of mathematical modeling research has been a subject of rapid development in these last years. We mention, for example, the mathematical model of drug therapy for chronic myeloid leukemia (CML) introduced in [20], or the hematopoietic system presented by Fokas et al. in [7] or, more recently the ODE-based model of CML treatment proposed by Moore and Li in [17].

The paper is divided into six sections. In Sect. 2 we consider a more abstract problem where the differential inclusion involves a perturbation guided by an operator $N: I \rightarrow C(C(I ; X) ; X)$

$$
u^{\prime \prime}(t) \in A(t) u(t)+F(t, N(t)(u)), t \in I
$$

and we introduce the concept of fundamental system and the definition of mild solution for a nonlocal problem. Next in Sect. 3 we list some properties of the solution multioperator in order to obtain the existence Theorem 3.5, which extends in a broad sense a recent theorem obtained by Henríquez et al. [11] for second order differential equations. We get the existence result through Martelli fixed point theorem of [15], by using the techniques for nonautonomous second order differential equations/inclusions developed by Kozak in [14], by Fattorini in [6] and by Cardinali-Gentili in [3]. 
Then, as consequence of Theorem 3.5, in Sect. 4 we are able to deduce the existence of mild solutions for the mentioned nonlocal problem (NLP) (see Theorem 4.1).

As application of our abstract result presented in Sect. 3, in Sect. 5 we obtain the controllability of a non-autonomous Cauchy problem guided by a wave equation, where the perturbation is subject to an opportune operator $N$.

Finally, Appendix contains some background material intended to make the paper self-contained.

\section{Problem Setting}

We consider the following nonlocal problem in a real Banach space $X$

$$
(N-N L P) \quad\left\{\begin{array}{l}
u^{\prime \prime}(t) \in A(t) u(t)+F(t, N(t)(u)), t \in I \\
u(0)=g(u) \\
u^{\prime}(0)=h(u),
\end{array}\right.
$$

where $\{A(t)\}_{t \in I}$ is a family of bounded linear operators $A(t): D(A) \rightarrow X$, where $D(A)$, independent on $t \in I$, is a subspace dense in $X$, generating a fundamental system $\{S(t, s)\}_{(t, s) \in I \times I}, F$ is a multimap and $N: I \rightarrow C(C(I ; X) ; X)$ is a map.

The concept of fundamental system, introduced by Kozak in [14], is recently used by Henríquez et al. [11] and by Cardinali, Gentili in [3].

In most of works, for every $t \in I$ the linear operator $A(t): D(A) \rightarrow X$ is also closed (see $[10,11,14]$ ), but we leave out this property on $A(t)$, since it is not necessary in order to obtain only the existence of mild solutions.

Definition 2.1. A family $\{S(t, s)\}_{(t, s) \in I \times I}$, where $S(t, s): X \rightarrow X$ is a bounded linear operator, is called fundamental system (or evolution system) generated by the family $\{A(t)\}_{t \in I}$ if

(S1) for each $x \in X$, the function $S(.,) x:. I \times I \rightarrow X$ is of class $C^{1}$ and

(a) for each $t \in I, S(t, t) x=0, \forall x \in X$;

(b) for each $t, s \in I$ and for each $x \in X,\left.\quad \frac{\partial S}{\partial t}(t, s)\right|_{t=s} x=x$ and $\frac{\partial S}{\partial s}(t$, $s)\left.\right|_{t=s} x=-x$;

(S2) for all $t, s \in I$, if $x \in D(A)$, then $S(t, s) x \in D(A)$ and the map $S(.,) x:. I \times I \rightarrow X$ is of class $C^{2}$. Moreover

(a) $\frac{\partial^{2} S}{\partial t^{2}}(t, s) x=A(t) S(t, s) x, \forall(t, s) \in I \times I, \forall x \in D(A)$;

(b) $\frac{\partial^{2} S}{\partial s^{2}}(t, s) x=S(t, s) A(s) x, \forall(t, s) \in I \times I, \forall x \in D(A)$;

(c) $\left.\frac{\partial^{2} S}{\partial s \partial t}(t, s)\right|_{t=s} x=0, \forall(t, s) \in I \times I, \forall x \in D(A)$;

(S3) for all $t, s \in I$, if $x \in D(A)$, then $\frac{\partial S}{\partial s}(t, s) x \in D(A)$. Moreover, there exist $\frac{\partial^{3} S}{\partial t^{2} \partial s}(t, s) x$ and $\frac{\partial^{3} S}{\partial s^{2} \partial t}(t, s) x$ such that
(a) $\frac{\partial^{3} S}{\partial t^{2} \partial s}(t, s) x=A(t) \frac{\partial S}{\partial s}(t, s) x, \forall(t, s) \in I \times I, \forall x \in D(A)$; 
(b) $\frac{\partial^{3} S}{\partial s^{2} \partial t}(t, s) x=\frac{\partial S}{\partial t}(t, s) A(s) x, \forall(t, s) \in I \times I, \forall x \in D(A) ; \quad$ and, for all $x \in D(A)$, the function $(t, s) \rightarrow A(t) \frac{\partial S}{\partial s}(t, s) x$ is continuous on $I \times I$.

As in [11], a map $S: I \times I \rightarrow \mathcal{L}(X)$, where $\mathcal{L}(X)$ denotes the space of all bounded linear operators in $X$ with the norm $\|\cdot\|_{\mathcal{L}(X)}$, is said to be a fundamental operator if $\{S(t, s)\}_{(t, s) \in I \times I}$ is a fundamental system. Moreover, for each $(t, s) \in I \times I$, we consider the operator

$$
C(t, s)=-\frac{\partial S}{\partial s}(t, s): X \rightarrow X
$$

and the family of linear operators $\{C(t, s)\}_{(t, s) \in I \times I}$.

Now we recall that by using Banach-Steinhaus Theorem there exist two constants $K, K_{1}>0$ such that (see [11])

$(P 0) \quad\|C(t, s)\|_{\mathcal{L}(X)} \leq K, \forall t, s \in I ;$

$(P 1) \quad\|S(t, s)\|_{\mathcal{L}(X)} \leq K|t-s|, \forall t, s \in I$;

$(P 2) \quad\|S(t, s)\|_{\mathcal{L}(X)} \leq K T, \forall t, s \in I$;

(P3) $\left\|S\left(t_{2}, s\right)-S\left(t_{1}, s\right)\right\|_{\mathcal{L}(X)} \leq K_{1}\left|t_{2}-t_{1}\right|, \forall t_{1}, t_{2}, s \in I$.

Further, if we assume that the fundamental system $\{S(t, s)\}_{(t, s) \in I \times I}$ has also the following property

(S4) $\forall x \in X, \frac{\partial^{2} S}{\partial t \partial s}(.,)$.$x is continuous on I \times I$,

we can claim that the family $\{C(t, s)\}_{(t, s) \in I \times I}$ satisfies

(P4) $\exists K_{2}>0:\left\|C\left(t_{2}, s\right)-C\left(t_{1}, s\right)\right\|_{\mathcal{L}(X)} \leq K_{2}\left|t_{1}-t_{2}\right|, \forall t_{1}, t_{2}, s \in I$.

For the sake of completeness we recall the fundamental Cauchy operator $G_{S}: L^{1}(I ; X) \rightarrow C(I ; X)$, introduced in [3] as

$$
G_{S} f(t)=\int_{0}^{t} S(t, s) f(s) d s, t \in I, f \in L^{1}(I ; X)
$$

and some its properties (see [3], Theorem 3.2 and Remark 3.3)

$$
\begin{aligned}
\left(G_{S} 1\right) & \left\|G_{S} f(t)-G_{S} g(t)\right\|_{X} \leq K T \int_{0}^{t}\|f(s)-g(s)\|_{X} d s, t \in I, f, g \in L^{1}(I ; X) ; \\
& \text { where } K T \text { is the constant presented in }(P 2) ; \\
\left(G_{S} 1\right)^{\prime} & \left\|G_{S} f-G_{S} g\right\|_{\infty} \leq K T\|f-g\|_{1}, \forall f, g \in L^{1}(I ; X) \\
\left(G_{S} 2\right) & \text { for any compact } H \subset X \text { and sequence }\left(f_{n}\right)_{n}, f_{n} \in L^{1}(I ; X) \text {, such that } \\
& \left\{f_{n}(t)\right\}_{n} \subset H, \text { for a.e. } t \in I, \text { the weak convergence } f_{n} \rightarrow \bar{f} \text { implies } \\
& \text { the convergence } G_{S} f_{n} \rightarrow G_{S} \bar{f} \text { in } C(I ; X) .
\end{aligned}
$$

In line with the definition of mild solution for the nonlocal problem presented in [11] for a non-autonomous second order equation, we introduce the following notion (see (2.1)). 
Definition 2.2. A continuous function $u: I \rightarrow X$ is said to be a mild solution for $(N-N L P)$ problem if

$$
u(t)=C(t, 0) g(u)+S(t, 0) h(u)+\int_{0}^{t} S(t, s) f(s) d s, t \in I
$$

where $f \in S_{F, N}^{1}(u)=\left\{f \in L^{1}(I ; X): f(t) \in F(t, N(t)(u))\right.$, a.e. $\left.t \in I\right\}$.

\section{Existence Result Under Perturbation with an Operator $N$}

\subsection{The Multioperator $S_{F, N}^{1}$}

First, we state the well-position of the superposition operator $S_{F, N}^{1}$.

Proposition 3.1. Let $X$ be a Banach space, $I$ be the closed interval $[0, T]$ and $F: I \times X \rightarrow \mathcal{P}_{k}(X)$ be a multimap which satisfies following hypotheses:

(F1) for every $x \in X, F(., x)$ has a strongly measurable selection;

(F2) for a.e. $t \in I, F(t,$.$) is upper semicontinuous on X$.

Let $N: I \rightarrow C(C(I ; X) ; X)$ be a map such that

(N1) for every $u \in C(I ; X), N().(u)$ is strongly measurable;

(N2) for every $u \in C(I ; X), N().(u)$ is bounded;

(FN) there exist $\alpha \in L_{+}^{1}(I)$ and a non-decreasing map $\psi: \mathbb{R}^{+} \rightarrow \mathbb{R}^{+}$such that

$$
\|F(t, N(t)(u))\| \leq \alpha(t) \psi\left(\|N(t)(u)\|_{X}\right), \text { a.e. } t \in I, \quad \forall u \in C(I ; X) .
$$

Then the multimap $S_{F, N}^{1}: C(I ; X) \rightarrow \mathcal{P}\left(L^{1}(I ; X)\right)$ given by

$$
S_{F, N}^{1}(u)=\left\{f \in L^{1}(I ; X): f(t) \in F(t, N(t)(u)) \text { a.e. } t \in I\right\}, \forall u \in C(I ; X)
$$

is well-defined.

Proof. We show that the multimap $S_{F, N}^{1}$ assumes non empty values. First of all, fixed $u \in C(I ; X)$, we define the following function $q_{u}: I \rightarrow X$, where

$$
q_{u}(t)=N(t)(u), \forall t \in I
$$

and by $(N 1)$ we say that $q_{u}$ is strongly measurable. Moreover, since the multimap $F$ has compact values and it satisfies $(F 1)$ and $(F 2)$, by applying Theorem 1.3 .5 of [13] there exists a strongly measurable selection $f_{u}: I \rightarrow X$ of the multimap $F\left(., q_{u}().\right)$. Further by $(F N)$ and by taking into account the boundedness of $N().(u)$ (see $(N 2)$ ), we have

$$
\left\|f_{u}(t)\right\|_{X} \leq\|F(t, N(t)(u))\| \leq \alpha(t) \psi\left(\sup _{t \in I}\|N(t)(u)\|_{X}\right) \doteq m_{u}(t) \text {, a.e. } t \in I,
$$

where it is easy to see that $m_{u} \in L_{+}^{1}(I)$. Hence $f_{u} \in L^{1}(I ; X)$, therefore $S_{F, N}^{1}(u) \neq \emptyset$ and so $S_{F, N}^{1}$ is well-defined. 
Remark 3.2. According to Kuratowski-Ryll-Nardzewski Theorem, if $X$ is a separable Banach space the property

$$
(F 1)^{\prime} \text { for every } x \in X, F(., x) \text { is measurable }
$$

is sufficient to have $(F 1)$ satisfied.

Let us note that in [4], sufficient conditions are given to obtain $(F 1)$ without the separability of the Banach space $X$.

Then we show some properties for the multioperator $S_{F, N}^{1}$.

Proposition 3.3. Let $X$ be a Banach space, $I=[0, T], F: I \times X \rightarrow \mathcal{P}_{k c}(X)$ and $N: I \rightarrow C(C(I ; X) ; X)$ be two maps satisfying all hypotheses of Proposition 3.1. If we consider the sequences $\left(u_{n}\right)_{n}, u_{n} \in C(I ; X)$ and $\left(f_{n}\right)_{n}$, $f_{n} \in S_{F, N}^{1}\left(u_{n}\right), n \in \mathbb{N}$, such that $u_{n} \rightarrow \bar{u}$ and $f_{n} \rightarrow \bar{f}$. Then $\bar{f} \in S_{F, N}^{1}(\bar{u})$.

Proof. Let us fix sequences $\left(u_{n}\right)_{n}, u_{n} \in C(I ; X)$, and $\left(f_{n}\right)_{n}, f_{n} \in S_{F, N}^{1}\left(u_{n}\right)$, such that

$$
u_{n} \rightarrow \bar{u} \text { in } C(I ; X) \text { and } f_{n} \rightarrow \bar{f} \text { in } L^{1}(I ; X) .
$$

According to Mazur's Theorem we have the existence of a double sequence of nonnegative numbers $\left(\alpha_{n, k}\right)_{n, k}$ such that

(I) $\forall n \in \mathbb{N}, \exists k_{0}(n) \in \mathbb{N}$ such that $\alpha_{n, k}=0, \forall k \geq k_{0}(n)$;

(II) $\sum_{k=n}^{\infty} \alpha_{n, k}=1, \forall n \in \mathbb{N}$;

(III) the following sequence $\left(\tilde{f}_{n}\right)_{n}$, where $\tilde{f}_{n}$ is defined by

$$
\tilde{f}_{n}(t)=\sum_{k=n}^{\infty} \alpha_{n, k} f_{k}(t), \forall t \in I,
$$

converges to $\bar{f}$ in the normed space $L^{1}(I ; X)$. Passing if necessary to a subsequence, we can assume that

$$
\left(\tilde{f}_{n}\right)_{n} \text { converges to } \bar{f} \text { a.e. on } I \text {. }
$$

Now, there exists $\widehat{N} \subset I, \mu(\widehat{N})=0$, such that for every $t \in I \backslash \widehat{N}$, we have (see $(F 2)$ and $(3.2))$ :

$\left(p_{1}\right) F(t,$.$) is upper semicontinuous at N(t)(\bar{u})$;

$\left(p_{2}\right) f_{n}(t) \in F\left(t, N(t)\left(u_{n}\right)\right), \forall n \in \mathbb{N}$;

$\left(p_{3}\right)\left(\tilde{f}_{n}(t)\right)_{n}$ converges to $\bar{f}(t)$,

where $\bar{u}$ and $\bar{f}$ are presented in (3.1).

The upper semicontinuity of $F(t,$.$) at N(t)(\bar{u}) \in X$ (see $\left.\left(p_{1}\right)\right)$ and the continuity of $N(t)($.$) at \bar{u} \in C(I ; X)$ imply, for every $\epsilon>0$, the existence of $W_{\epsilon}(F(t, N(t)(\bar{u})))$, an $\epsilon$-neighborhood of the set $F(t, N(t)(\bar{u}))$, and of $\bar{n}=$ $\bar{n}(\epsilon, t, \bar{u}) \in \mathbb{N}$ such that 


$$
F\left(t, N(t)\left(u_{n}\right)\right) \subset W_{\epsilon}(F(t, N(t)(\bar{u}))), \forall n \geq \bar{n} .
$$

Next, by $\left(p_{2}\right)$ we can write

$$
f_{n}(t) \in F\left(t, N(t)\left(u_{n}\right)\right) \subset W_{\epsilon}(F(t, N(t)(\bar{u}))), \forall n \geq \bar{n} .
$$

Then the convexity of the $\epsilon$-neighborhood $W_{\epsilon}(F(t, N(t)(\bar{u})))$ and (3.3) imply that

$$
\tilde{f}_{n}(t) \in W_{\epsilon}(F(t, N(t)(\bar{u}))), \forall n \geq \bar{n}
$$

is true. Hence by $\left(p_{3}\right)$ we have

$$
\bar{f}(t) \in \overline{W_{\epsilon}(F(t, N(t)(\bar{u})))} .
$$

Now, by the arbitrariness of $\epsilon>0$ and by recalling that the set $F(t, N(t)(\bar{u}))$ is closed, we obtain

$$
\bar{f}(t) \in F(t, N(t)(\bar{u})) .
$$

Finally, since (3.4) is true a.e. on $I$ and that $\bar{f} \in L^{1}(I ; X)$, we conclude that $\bar{f} \in S_{F, N}^{1}(\bar{u})$.

\subsection{The Solution Multioperator}

Now, in order to establish some properties of the solution multioperator, we assume the following property on the family $\{A(t)\}_{t \in I}$ :

$(A)^{\prime}\{A(t)\}_{t \in I}$ is a family of bounded linear operators, defined in a subspace $D(A)$ dense in $X$ and taking values in $X$ such that, for each $x \in D(A)$, the function $t \rightarrow A(t) x$ is continuous on $I$ and generating a fundamental system $\{S(t, s)\}_{(t, s) \in I \times I}$ where, for each $(t, s) \in I \times I, S(t, s)$ is compact (i.e. it is continuous and it maps bounded sets into relatively compact sets).

Proposition 3.4. Let $X$ be a separable Banach space, $I=[0, T],\{A(t)\}_{t \in I}$ be a family with property $(A)^{\prime}$.

Let $F: I \times X \rightarrow \mathcal{P}_{k c}(X)$ be a map which satisfies hypotheses $(F 1)$ and (F2) of Proposition 3.1 and the following property:

(F3) there exists $m \in L_{+}^{1}(I): \eta(F(t, B)) \leq m(t) \eta(B)$, for a.e. $t \in I$ and for every bounded set $B \subset X$ (where $\eta$ is the Hausdorff $M N C$ in $X$ ).

Let $N: I \rightarrow C(C(I ; X) ; X)$ be a map which has properties $(N 1)$ and $(F N)$ of Proposition 3.1 and the following

$(N 2)^{\prime}$ for every bounded $W \subset C(I ; X)$, the set $\{N(t)(u): t \in I, u \in W\}$ is bounded.

Let $g, h: C(I ; X) \rightarrow X$ be two functions which satisfy following properties:

(gh1) $g, h$ are compact; 
(gh2) $g, h$ are bounded.

Then the solution multioperator $\Gamma: C(I ; X) \rightarrow \mathcal{P}(C(I ; X))$ defined as

$$
\begin{aligned}
\Gamma(u)=\{v & \in C(I ; X): v(t)=C(t, 0) g(u)+S(t, 0) h(u)+ \\
& \left.+\int_{0}^{t} S(t, s) f(s) d s, t \in I, f \in S_{F, N}^{1}(u)\right\}, u \in C(I ; X)
\end{aligned}
$$

has nonempty closed convex values. Moreover $\Gamma$ is totally bounded and upper semicontinuous.

Proof. First, for all $u \in C(I ; X)$, Proposition 3.1 implies that there exists $f \in S_{F, N}^{1}(u)$. Now, the function $v: I \rightarrow X$, where

$$
v(t)=C(t, 0) g(u)+S(t, 0) h(u)+\int_{0}^{t} S(t, s) f(s) d s, t \in I
$$

is well-defined, since for every $t \in I$, the map $S(t,) f.($.$) is B-integrable on$ $[0, t]$. Indeed, put $p_{t}:[0, t] \times X \rightarrow X$, where $p_{t}(s, x)=S(t, s) x$, for every $(s, x) \in[0, t] \times X$, property $(S 1)$ of the fundamental system implies that the map $p_{t}(., f())=.S(t,) f.($.$) is strongly measurable (see [13], Theorem 1.3.5)$ and by $(P 2)$ it is also B-integrable on $[0, t]$. Moreover $v$ is also continuous on $I$, so $v \in \Gamma(u)$, i.e. $\Gamma(u) \neq \emptyset$. Finally $\Gamma$ takes convex values thanks to the convexity of the values of $F$.

From now on we proceed by steps.

Step 1: The solution multioperator $\Gamma$ has closed graph (therefore $\Gamma$ has also closed values).

Let $\left(u_{n}\right)_{n}$ be a sequence in $C(I ; X)$ such that $u_{n} \rightarrow u^{*}$ in $C(I ; X)$ and $\left(v_{n}\right)_{n}$ be a sequence in $C(I ; X), v_{n} \in \Gamma\left(u_{n}\right), \forall n \in \mathbb{N}$, with $v_{n} \rightarrow v^{*}$ in $C(I ; X)$. We show that $v^{*} \in \Gamma\left(u^{*}\right)$.

Since for every $n \in \mathbb{N}, v_{n} \in \Gamma\left(u_{n}\right)$, there exists $f_{n} \in S_{F, N}^{1}\left(u_{n}\right)$ such that

$$
v_{n}(t)=C(t, 0) g\left(u_{n}\right)+S(t, 0) h\left(u_{n}\right)+\int_{0}^{t} S(t, s) f_{n}(s) d s, t \in I .
$$

We will prove that there exists $f^{*} \in S_{F, N}^{1}\left(u^{*}\right)$ such that

$$
v^{*}(t)=C(t, 0) g\left(u^{*}\right)+S(t, 0) h\left(u^{*}\right)+\int_{0}^{t} S(t, s) f^{*}(s) d s, t \in I .
$$

Fixed $t \in I$, since $C(t, 0), S(t, 0) \in \mathcal{L}(X)$ and $g, h$ are continuous in $u^{*}$, it follows that for $n \rightarrow \infty$ the following convergences 


$$
\begin{aligned}
& C(t, 0) g\left(u_{n}\right) \rightarrow C(t, 0) g\left(u^{*}\right), \\
& S(t, 0) h\left(u_{n}\right) \rightarrow S(t, 0) h\left(u^{*}\right)
\end{aligned}
$$

are true. Now we deduce that the set $\left\{f_{n}\right\}_{n}$ is integrably bounded. Indeed, since

$$
W=\left\{u_{n}, u^{*}: n \in \mathbb{N}\right\}
$$

is bounded, hypothesis $(N 2)^{\prime}$ implies that the set $\{N(t)(u): t \in I, u \in W\}$ is also bounded. Then, by using $(F N)$, we have

$$
\left\|f_{n}(t)\right\|_{X} \leq \alpha(t) \psi\left(\sup _{\substack{t \in I \\ u \in W}}\|N(t)(u)\|\right) \doteq \alpha(t) P,
$$

where by $(N 2)^{\prime}$ the constant $P$ is not depending on $t$. Therefore $\left\{f_{n}\right\}_{n}$ is integrably bounded.

Furthermore, let us show that the set $\left\{f_{n}(t)\right\}_{n}$ is relatively compact in $X$ for a.e. $t \in I$.

Indeed, by using (F3) and the monotonicity of the Hausdorff MNC $\eta$, for a.e. $t \in I$, being the set $\left\{N(t)\left(u_{n}\right)\right\}_{n} \in \mathcal{P}_{b}(X)$, according to $(N 2)^{\prime}$ we estimate

$$
0 \leq \eta\left(\left\{f_{n}(t)\right\}_{n}\right) \leq \eta\left(F\left(t,\left\{N(t)\left(u_{n}\right)\right\}_{n}\right)\right) \leq m(t) \eta\left(\left\{N(t)\left(u_{n}\right)\right\}_{n}\right) .
$$

Since the map $N(t) \in C(C(I ; X) ; X)$, the compactness of $W$ (see (3.8)) implies that also $N(t)(W)$ is compact. Hence by (3.9) we have

$$
0 \leq \eta\left(\left\{f_{n}(t)\right\}_{n}\right) \leq m(t) \eta\left(\left\{N(t)\left(u_{n}\right)\right\}_{n}\right) \leq m(t) \eta(N(t)(W))=0,
$$

therefore $\eta\left(\left\{f_{n}(t)\right\}_{n}\right)=0$, i.e. the set $\left\{f_{n}(t)\right\}_{n}$ is relatively compact in $X$.

Now, being the sequence $\left(f_{n}\right)_{n}$ semicompact, we can use Proposition 4.2.1 of [13] to conclude that the set $\left\{f_{n}\right\}_{n}$ is weakly compact in $L^{1}(I ; X)$, so w.l.o.g. there exists $f^{*} \in L^{1}(I ; X)$ such that $f_{n} \rightarrow f^{*}$ in $L^{1}(I ; X)$.

Hence, since the fundamental Cauchy operator $G_{S}$ satisfies the mentioned properties $\left(G_{S} 1\right)^{\prime}$ and $\left(G_{S} 2\right)$, we are in a position to apply Theorem 5.1.1 of [13] and we deduce

$$
G_{S} f_{n} \rightarrow G_{S} f^{*} \text { in } C(I ; X) .
$$

Consequently, by passing to the limit in (3.5), properties (3.6, (3.7) and (3.10)) imply the following

$$
\lim _{n \rightarrow \infty} v_{n}(t)=C(t, 0) g\left(u^{*}\right)+S(t, 0) h\left(u^{*}\right)+G_{S} f^{*}(t), t \in I
$$


and, by recalling that $v_{n} \rightarrow v^{*}$ in $C(I ; X)$, the uniqueness of the limit algorithm guarantees that

$$
v^{*}(t)=C(t, 0) g\left(u^{*}\right)+S(t, 0) h\left(u^{*}\right)+\int_{0}^{t} S(t, s) f^{*}(s) d s, t \in I .
$$

Finally, according to Proposition 3.3 we have that $f^{*} \in S_{F, N}^{1}\left(u^{*}\right)$. Hence we can conclude that $v^{*} \in \Gamma\left(u^{*}\right)$, therefore $\Gamma$ has closed graph.

Step 2: $\Gamma$ is totally bounded.

We fix a bounded subset $\Omega$ of $C(I ; X)$.

Step 2a: First we show that $\Gamma(\Omega)$ is equicontinuous on $I$.

To prove this, fixed $v \in \Gamma(\Omega)$, let be $u_{v} \in \Omega$ such that $v \in \Gamma\left(u_{v}\right)$, and $f \in S_{F ; N}^{1}\left(u_{v}\right)$. By using $(P 4),(P 3),(g h 2),(P 2),(F N)$ and $(N 2)^{\prime}$, let us note that, for every $t_{1}, t_{2} \in I$, w.l.o.g. $t_{1} \leq t_{2}$, we have

$$
\begin{aligned}
\| v\left(t_{2}\right)- & v\left(t_{1}\right)\left\|_{X} \leq K_{2}\left|t_{2}-t_{1}\right|\right\| g\left(u_{v}\right)\left\|_{X}+K_{1}\left|t_{2}-t_{1}\right|\right\| h\left(u_{v}\right) \|_{X} \\
& +\int_{t_{1}}^{t_{2}}\left\|S\left(t_{2}, s\right)\right\|_{\mathcal{L}(X)}\|f(s)\|_{X} d s+\int_{0}^{t_{1}}\left\|S\left(t_{2}, s\right)-S\left(t_{1}, s\right)\right\|_{\mathcal{L}(X)}\|f(s)\|_{X} d s, \\
\leq & K_{2}\left|t_{2}-t_{1}\right| G+K_{1}\left|t_{2}-t_{1}\right| H \\
& +\int_{t_{1}}^{t_{2}} K T\left\|F\left(s, N(s)\left(u_{v}\right)\right)\right\| d s+\int_{0}^{t_{1}} K_{1}\left|t_{2}-t_{1}\right|\left\|\left(s, N(s)\left(u_{v}\right)\right)\right\| d s \\
\leq & \left(K_{2} G+K_{1} H\right)\left|t_{2}-t_{1}\right|+K T\|\alpha\|_{1} P_{\Omega}\left|t_{2}-t_{1}\right|+K_{1}\|\alpha\|_{1} P_{\Omega}\left|t_{2}-t_{1}\right| \\
\leq & C\left|t_{2}-t_{1}\right|,
\end{aligned}
$$

where $G, H, P_{\Omega}$ and $C$ are respectively the positive constants so defined:

$$
\begin{aligned}
G & =\sup _{u \in C(I ; X)}\|g(u)\|_{X}, \\
H & =\sup _{u \in C(I ; X)}\|h(u)\|_{X} \\
P_{\Omega} & =\psi\left(\sup _{\substack{s \in I \\
u \in \Omega}}\|N(s)(u)\|_{X}\right), \\
C & =\max \left\{K_{2} G ; K_{1} H ; K T\|\alpha\|_{1} P_{\Omega} ; K_{1}\|\alpha\|_{1} P_{\Omega}\right\} .
\end{aligned}
$$

Obviously this inequality also holds if $t_{1}>t_{2}$.

So the set $\Gamma(\Omega)$ is equilipschitzian and therefore it is also equicontinuous on $I$.

Step 2b: Next we prove that $\Gamma(\Omega)(t)$ is relatively compact in $X$, for every $t \in I$.

To this end, we consider the set 


$$
S_{\Omega}=\left\{f \in S_{F, N}^{1}(u), u \in \Omega\right\} .
$$

Our first goal is to show that hypotheses of Theorem 5.23 of [12] are satisfied in order to prove the relative compactness of the set

$$
H_{\Omega}(t)=\left\{\int_{0}^{t} S(t, s) f(s) d s, f \in S_{\Omega}\right\},
$$

for every $t \in I$.

Being $X$ a separable Banach space, the space $L^{1}(I ; X)$ is separable too. Hence the closed set $\bar{S}_{\Omega}{ }^{1}$ is separable. Therefore there exists a countable set $\left\{f_{n}\right\}_{n} \subset S_{\Omega}$ such that

$$
{\overline{\left\{f_{n}\right\}_{n}}}^{L^{1}}={\overline{S_{\Omega}}}^{L^{1}} .
$$

In a preliminary way, let us define the multimap $G: I \rightarrow \mathcal{P}(X)$ in the following way

$$
G(t)=\overline{c o}\left\{f_{n}(t): n \in \mathbb{N}\right\}, t \in I .
$$

Now, we obtain that $G$ is measurable. By the completeness and the separability of the Banach space $X$, it is enough to prove that (see [12], Proposition 2.3) there exists a countable set $E$ of measurable selectors of $G$ such that

$$
G(t)=\overline{E(t)}, t \in I \text {. }
$$

To this aim, we define the set of functions

$$
E=\left\{\gamma: I \rightarrow X: \gamma(t)=\sum_{n=1}^{+\infty} q_{n} f_{n}(t), t \in I,\left(q_{n}\right)_{n} \in Q\right\}
$$

where $Q$ is the countable set of non negative rational sequences so defined

$$
Q=\left\{\left(q_{n}\right)_{n}: \sum_{n=1}^{+\infty} q_{n}=1, q_{n} \in \mathbb{Q}_{0}^{+}, \exists k \in \mathbb{N}: q_{n}=0, n>k\right\} .
$$

Clearly, the countability of $Q$ implies that the set $E$ is countable too. Moreover, let us note that every $\gamma \in E$ is a measurable selector of $G$ (see (3.13)).

Now, fixed $t \in I$, we prove that (3.14) holds.

By (3.15) and (3.13) obviously we can write

$$
\overline{E(t)} \subset G(t) .
$$

To get the other inclusion it is sufficient to prove that 


$$
c o\left\{f_{n}(t): n \in \mathbb{N}\right\} \subset \overline{E(t)} .
$$

Let us fix $p \in \operatorname{co}\left\{f_{n}(t): n \in \mathbb{N}\right\}$, i.e. $p=\sum_{n=1}^{+\infty} \alpha_{n} f_{n}(t)$, where $\alpha_{n} \in[0,1]$, $\sum_{n=1}^{+\infty} \alpha_{n}=1$ and there exists $k \in \mathbb{N}$ such that $\alpha_{n}=0, n>k$.

Obviously we have that there exist $k$ sequences $\left(q_{m}^{1}\right)_{m}, \ldots,\left(q_{m}^{k}\right)_{m}, q_{m}^{i} \in$ $[0,1] \cap Q, i=1, \ldots, k$ and $m \in \mathbb{N}$, converging respectively to $\alpha_{1}, \ldots, \alpha_{k}$.

So $p=\lim _{m \rightarrow+\infty}\left(\sum_{i=1}^{k} q_{m}^{i} f_{i}(t)\right) \in \overline{E(t)}$. Therefore (3.16) is true. surable.

Then, being (3.14) proved, we can conclude that the multimap $G$ is mea-

Next we show that $G$ is integrably bounded.

First of all, if $u_{n} \in \Omega$ is such that $f_{n} \in S_{F, N}^{1}\left(u_{n}\right)$, for every $n \in \mathbb{N}$, from (3.13), by recalling hypotheses $(F N)$ and $(N 2)^{\prime}$, for every $n \in \mathbb{N}$, we can write the following inequality

$$
\left\|F\left(t, N(t)\left(u_{n}\right)\right)\right\| \leq \alpha(t) P_{\Omega} \doteq \omega_{\Omega}(t) \text {, a.e. } t \in I
$$

where $P_{\Omega}=\psi\left(\sup _{\substack{t \in I \\ u \in \Omega}}\|N(t)(u)\|_{X}\right) \in \mathbb{R}_{0}^{+}$. So we can deduce

$$
\|G(t)\| \leq\left\|\overline{c o}\left\{F\left(t, N(t)\left(u_{n}\right)\right): n \in \mathbb{N}\right\}\right\| \leq \omega_{\Omega}(t), \text { a.e. } t \in I
$$

and being $\omega_{\Omega} \in L_{+}^{1}(I)$, we can conclude that $G$ is integrably bounded.

Now, put $\widetilde{N}=\left\{\sigma \in[0, t]:\|G(\sigma)\|>\omega_{\Omega}(\sigma)\right\}$ (see (3.17)), for every $t \in I$ we consider the multimap $\Phi_{t}:[0, t] \rightarrow \mathcal{P}(X)$ so defined

$$
\Phi_{t}(s)=\left\{\begin{array}{l}
\overline{S(t, s) G(s)}, s \in[0, t] \backslash \widetilde{N} \\
\{0\}, s \in[0, t] \cap \widetilde{N} .
\end{array}\right.
$$

From (3.17) and by taking into account $(A)^{\prime}$, it is easy to say that, for every $s \in[0, t]$, the set $\Phi_{t}(s)$ is compact and convex.

Moreover, by combining (3.17) with $(P 2)$, we have that $\Phi_{t}$ is integrably bounded.

Now we shall prove that $\Phi_{t}$ is graph measurable.

Again in virtue of the separability of $X$, it is enough to prove that $\Phi_{t}$ is measurable (see [12], Proposition 1.7).

To this aim we define the following multimap:

$$
\begin{aligned}
G_{t}^{*}:[0, t] \times X & \rightarrow \mathcal{P}(X) \\
(s, x) & \rightarrow G_{t}^{*}(s, x) \doteq G(s)
\end{aligned}
$$

and we note that the measurability of $G$ implies that $G_{t}^{*}$ is measurable too.

Next, since for every $s \in[0, t], G_{t}^{*}(s,$.$) is obviously lower semicontinuous$ on the separable Banach space $X$ and by applying Proposition 7.17 of [12], we deduce that $G_{t}^{*}$ is (S.D.)-lower semicontinuous. 
Now, in a preliminary way, we consider the multimap $\Phi_{t}^{*}:[0, t] \times X \rightarrow$ $\mathcal{P}(X)$ defined by

$$
\Phi_{t}^{*}(s, x)=S(t, s) G(s)=S(t, s) G_{t}^{*}(s, x), \forall(s, x) \in[0, t] \times X
$$

and we show that $\Phi_{t}^{*}$ is (S.D.)-lower semicontinuous.

In fact $G_{t}^{*}$ is (S.D.)-l.s.c. and hence for every $\epsilon>0$, there exists a closed set $T_{\epsilon} \subset[0, t]: \mu\left([0, t] \backslash T_{\epsilon}\right)<\epsilon$ such that the restriction of $G_{t}^{*}$ to $T_{\epsilon} \times X$ is lower semicontinuous. So we can deduce that $\Phi_{t}^{*}$ is l.s.c. on $T_{\epsilon} \times X$.

Indeed let us fix $(\bar{s}, \bar{x}) \in T_{\epsilon} \times X$, a net $\left\{\left(s_{\alpha}, x_{\alpha}\right)\right\}_{\alpha \in J}$ such that

$$
\left(s_{\alpha}, x_{\alpha}\right) \rightarrow(\bar{s}, \bar{x}) \text { in } \mathbb{R} \times X
$$

and a point $\bar{y} \in \Phi_{t}^{*}(\bar{s}, \bar{x})$. We can find $\bar{z} \in G_{t}^{*}(\bar{s}, \bar{x})$ such that $\bar{y}=S(t, \bar{s}) \bar{z}$ (see $(3.19))$. Then since $G_{t}^{*}$ is l.s.c. at $(\bar{s}, \bar{x})$, from Proposition 2.6 of [12] there exists a net $\left(z_{\alpha}\right)_{\alpha \in J}, z_{\alpha} \in G_{t}^{*}\left(s_{\alpha}, x_{\alpha}\right), \alpha \in J$, such that

$$
z_{\alpha} \rightarrow \bar{z} \text { in } X
$$

Next, since this estimate is true

$$
\left\|S\left(t, s_{\alpha}\right) z_{\alpha}-\bar{y}\right\|_{X} \leq K T\left\|z_{\alpha}-\bar{z}\right\|_{X}+\left\|S\left(t, s_{\alpha}\right)-S(t, \bar{s})\right\|_{\mathcal{L}(X)}\|\bar{z}\|_{X},
$$

by $(A)^{\prime}$ and $(3.20)$, we can conclude that

$$
S\left(t, s_{\alpha}\right) z_{\alpha} \rightarrow \bar{y} \text { in } X \text {. }
$$

Then, according to Proposition 2.6 of [12], $\Phi_{t}^{*}$ is l.s.c. on $T_{\epsilon} \times X$.

Further by Proposition 2.38 of [12] we deduce that also the closed multimap $\overline{\Phi_{t}^{*}}$ is (S.D.)-l.s.c. .

Moreover fixed $s \in[0, t]$, being $\overline{\Phi_{t}^{*}(s, .)}=\overline{S(t, s) G(s)}$ constant on $X$, $\overline{\Phi_{t}^{*}(s, .)}$ is also l.s.c. on the separable Banach space $X$. Therefore, from Theorem 3.2 of [19] we can say that $\overline{\Phi_{t}^{*}}$ is $\mathcal{M}(\mathbb{R}) \times \mathcal{B}(X)$-measurable. Therefore it is easy to see that the multimap $\Phi_{t}$, defined in (3.18), is measurable too.

Hence we can invoke Proposition 1.7 of [12] in order to say that $\Phi_{t}$ is graph measurable.

Finally, by using the proof of Theorem 5.23 of [12] we can get

$$
\int_{0}^{t} \Phi_{t}(s) d s \in \mathcal{P}_{k c}(X) .
$$

Now, let us note that the following inclusion

$$
H_{\Omega}(t) \subset \int_{0}^{t} \Phi_{t}(s) d s
$$

holds. 
To this end let us fix $\int_{0}^{t} S(t, s) f(s) d s \in H_{\Omega}(t)$ (see (3.11)). By (3.12) we deduce that there exists a subsequence $\left(f_{n_{k}}\right)_{k}$ of $\left(f_{n}\right)_{n}$ :

$$
f_{n_{k}} \rightarrow f \text { in } L^{1}(I ; X)
$$

Obviously Riesz Theorem implies that (see (3.13))

$$
f(s)=\lim _{n_{k} \rightarrow \infty} f_{n_{k}}(s) \in G(s) \text { a.e. } s \in I,
$$

hence $f \in S_{G}^{1}$. Then we have (see (3.18))

$$
\int_{0}^{t} S(t, s) f(s) d s \subset \int_{0}^{t} \overline{S(t, s) G(s)} d s=\int_{0}^{t} \Phi_{t}(s) d s .
$$

Therefore we can conclude that (3.22) is satisfied and so, by (3.21) we have that the set $H_{\Omega}(t)$ is relatively compact. Now we note that

$$
\Gamma(\Omega)(t) \subset C(t, 0) g(\Omega)+S(t, 0) h(\Omega)+H_{\Omega}(t) .
$$

Then, by taking into account $(g h 1)$ and the continuity of operators $C(t, 0)$ and $S(t, 0)$, we can conclude that the set $\Gamma(\Omega)(t)$ is relatively compact in $X$.

So, bearing in mind also the equicontinuity of $\Gamma(\Omega)$, we have that $\Gamma(\Omega)$ is relatively compact in $C(I ; X)$. Therefore the multimap $\Gamma$ is totally bounded, hence $\Gamma$ is also locally compact. Now, by recalling that $\Gamma$ has closed graph, we can use Proposition 4.1.16 of [5] and deduce that $\Gamma$ is upper semicontinuous in $C(I ; X)$.

\subsection{Main Abstract Existence Result}

Finally we are in a position to prove the existence at least of a mild solution for the nonlocal problem $(N-N L P)$.

Theorem 3.5. Let $X$ be a separable Banach space, $I=[0, T],\{A(t)\}_{t \in I}$ be a family with property $(A)^{\prime}$.

Let $F: I \times X \rightarrow \mathcal{P}_{k c}(X)$ be a map which satisfies $(F 1),(F 2)$, (F3) of Proposition 3.4 and $N: I \rightarrow C(C(I ; X) ; X)$ be a map with properties $(N 1),(F N)$ of Proposition 3.1 and such that

$(N 2)_{s} N$ is bounded.

Moreover let $g, h: C(I ; X) \rightarrow X$ be two functions as in Proposition 3.4.

Then there exists at least one mild solution for the nonlocal problem $(N-N L P)$.

Proof. We consider the same solution multioperator $\Gamma$ of Proposition 3.4:

$$
\Gamma(u)=\{v \in C(I ; X): v(t)=C(t, 0) g(u)+S(t, 0) h(u)+
$$




$$
\left.+\int_{0}^{t} S(t, s) f(s) d s, t \in I, f \in S_{F, N}^{1}(u)\right\}, u \in C(I ; X) .
$$

In the setting of our hypotheses we are in a position to use Proposition 3.4 and so we can say that $\Gamma$ has nonempty closed convex values, it is totally bounded and upper semicontinuous. Now, in order to prove the existence of a fixed point for the multioperator $\Gamma$, we show that the set

$$
\Omega=\{u \in C(I ; X): \exists \lambda>1 \text { such that } \lambda u \in \Gamma(u)\}
$$

is bounded.

Let us fix $u \in \Omega$, then there exists $\lambda>1$ such that $\lambda u \in \Gamma(u)$ and $f_{u} \in S_{F, N}^{1}(u)$. At this point we observe that, by using $(P 2),(F N),(N 2)_{s}$ and $(g h 2)$, we obtain

$$
\begin{aligned}
\|u(t)\|_{X} \leq & \|C(t, 0)\|_{\mathcal{L}(X)}\|g(u)\|_{X}+\|S(t, 0)\|_{\mathcal{L}(X)}\|h(u)\|_{X}+ \\
& +\int_{0}^{t}\|S(t, s)\|_{\mathcal{L}(X)}\|F(s, N(s)(u))\| d s \\
\leq & K G+K T H+K T \psi(M)\|\alpha\|_{1} \doteq C, t \in I
\end{aligned}
$$

where $\|g\|_{\infty} \leq G,\|h\|_{\infty} \leq H$ and $\|N(t)(u)\|_{X} \leq M, \forall t \in I, \forall u \in C(I ; X)$. Hence we can conclude that the set $\Omega$ is bounded in $C(I ; X)$.

Finally, we are ready to proceed to the application of Theorem 6.1 and we have the existence at least of one fixed point for the solution multioperator $\Gamma$, i.e. there exists at least one mild solution for $(N-N L P)$ problem.

Remark 3.6. Let us note that $(N-N L P)$ problem can be rewritten as the following problem

$$
(N L P)^{*}\left\{\begin{array}{l}
u^{\prime \prime}(t) \in A(t) u(t)+F(t, u(t)), t \in I \\
u(0)=g(u) \\
u^{\prime}(0)=h(u),
\end{array}\right.
$$

studied in [3], by considering the map $N: I \rightarrow C(C(I ; X) ; X)$ so defined

$$
N(t)(u)=u(t), \forall t \in I, \forall u \in C(I ; X) .
$$

Unfortunately we note that Theorem 3.5 does not allow to prove the existence of mild solutions for $(N L P)^{*}$ problem because $N$ does not satisfy $(N 2)_{s}$ of Theorem 3.5.

Remark 3.7. Clearly if $g$ and $h$ are two constant functions, our Theorem 3.5 is an existence proposition for a Cauchy problem, where (gh1) and (gh2) obviously hold. 


\section{Existence Result with a Stabilizing Effect}

Now, as a consequence of Theorem 3.5, we are in a position to provide a result for the nonlocal problem $(N L P)$.

Theorem 4.1. Let $X$ be a separable Banach space, $I=[0, T],\{A(t)\}_{t \in I}$ be a family which satisfies $(A)^{\prime}$ and $R>0$.

Let $F: I \times X \rightarrow \mathcal{P}_{k c}(X)$ be a map with following hypotheses:

(F1) for every $x \in X, F(., x)$ has a strongly measurable selection;

$(F 2)$ for a.e. $t \in I, F(t,$.$) is upper semicontinuous on X$;

(F3) there exists $m \in L_{+}^{1}(I): \eta(F(t, B)) \leq m(t) \eta(B)$,

for a.e. $t \in I$ and for every bounded set $B \subset X$

(where $\eta$ is the Hausdorff $M N C$ in $X$ );

(F4) there exist $\alpha \in L_{+}^{1}(I)$ and a non-decreasing map $\psi: \mathbb{R}^{+} \rightarrow \mathbb{R}^{+}$such that

$$
\|F(t, x)\| \leq \alpha(t) \psi\left(\|x\|_{X}\right) \text {, a.e. } t \in I, \forall x \in \overline{B_{X}(0, R)} .
$$

Let $g, h: C(I ; X) \rightarrow X$ be two functions which satisfy following properties:

$$
\begin{aligned}
& \text { (gh1) } g, h \text { are compact; } \\
& \text { (gh2) } g, h \text { are bounded. }
\end{aligned}
$$

Then there exists at least one mild solution for the nonlocal problem (NLP).

Proof. First, fixed $R>0$, we note that we can define the map $N: I \rightarrow$ $C(C(I ; X) ; X)$ in the following way: for every $t \in I, N(t): C(I ; X) \rightarrow X$ is such that

$$
N(t)(u) \doteq u_{R}(t), \forall u \in C(I ; X) .
$$

Next we observe that for every $u \in C(I ; X)$, we have that $N().(u)=u_{R}($.$) is$ continuous on $I$, so $N().(u)$ is also strongly measurable and moreover obviously $\|N(t)(v)\|_{X} \leq R, \forall t \in I$ and $\forall v \in C(I ; X)$. Therefore $N$ satisfies $(N 1)$ and $(N 2)_{s}$ of Theorem 3.5. Further, for every $u \in C(I ; X)$, since $\left\|u_{R}(t)\right\|_{X} \leq R$, by combining $(F 4)$ with $(4.1)$, we have the following

$$
\begin{aligned}
\|F(t, N(t)(u))\| & =\left\|F\left(t, u_{R}(t)\right)\right\| \leq \\
& \leq \alpha(t) \psi\left(\left\|u_{R}(t)\right\|_{X}\right)=\alpha(t) \psi\left(\|N(t)(u)\|_{X}\right), \text { a.e. } t \in I,
\end{aligned}
$$

so $(F N)$ holds.

Therefore Theorem 3.5 establishes the existence of at least one mild solution for the nonlocal problem $(N L P)$.

Remark 4.2. Let us observe that it is not possible to compare our Theorem 4.1 with Theorem 4.1 of [3], since two studied problems are different. In fact in 
$(N L P)$ problem, we have a stabilization of the effect of the perturbation on the mild solution outside the ball $\overline{B_{X}(0, R)}$.

\section{An Application: Controllability with Stabilizing Effect}

In this section we apply the theory developed in Sect. 3 to study the following controllability problem

$$
\left\{\begin{array}{l}
\frac{\partial^{2} w}{\partial t^{2}}(t, \xi)=\frac{\partial^{2} w}{\partial \xi^{2}}(t, \xi)+b(t) \frac{\partial w}{\partial \xi}(t, \xi)+f\left(t, \min \left\{1 ; \max _{s \in I}\|w(s, .)\|_{L^{2}}\right\} \int_{0}^{t} p(s) d s\right)+u(t, \xi) \\
w(t, 0)=w(t, 2 \pi), t \in I \\
\frac{\partial w}{\partial \xi}(t, 0)=\frac{\partial w}{\partial \xi}(t, 2 \pi), t \in I \\
w(0, \xi)=x_{0}, \xi \in \mathbb{R} \\
\frac{\partial w}{\partial t}(0, \xi)=x_{1}, \xi \in \mathbb{R} \\
u(t, \xi) \in\left[\varphi_{1}(t), \varphi_{2}(t)\right] \doteq\left\{\lambda \varphi_{1}(t)+(1-\lambda) \varphi_{2}(t), \lambda \in[0,1]\right\}, t \in I, \xi \in \mathbb{R}
\end{array}\right.
$$

where $x_{0}, x_{1} \in \mathbb{C}, I=[0, T], T>0$ and $f: I \times \mathbb{C} \rightarrow \mathbb{C}, b: I \rightarrow \mathbb{R}, p: I \rightarrow \mathbb{C}$, $\varphi_{1}, \varphi_{2}: I \rightarrow \mathbb{C}$ are suitable functions.

The following application shows a situation in which our Theorem 3.5 works, but Theorem 4.1 of [3] not.

First, as in [11], we will use the identification between functions defined on the quotient group $\mathbb{T}=\mathbb{R} / 2 \pi \mathbb{Z}$ with values in $\mathbb{C}$, and $2 \pi$-periodic functions from $\mathbb{R}$ to $\mathbb{C}$. In order to model the problem above in an abstract form, we consider the space $X=L^{2}(\mathbb{T} ; \mathbb{C})$, i.e. the space of all functions $x: \mathbb{R} \rightarrow \mathbb{C}$, $2 \pi$-periodic and 2 -integrable in $[0,2 \pi]$, endowed with the usual norm $\|\cdot\|_{2}$. Moreover we denote by $H^{1}(\mathbb{T}, \mathbb{C})$ and by $H^{2}(\mathbb{T} ; \mathbb{C})$ respectively the following subspaces of $L^{2}(\mathbb{T} ; \mathbb{C})$

$$
\begin{gathered}
H^{1}(\mathbb{T} ; \mathbb{C})=\left\{x: \mathbb{R} \rightarrow \mathbb{C}, 2 \pi-\text { periodic, } x^{\prime} \in L^{2}(\mathbb{T} ; \mathbb{C})\right\} \\
H^{2}(\mathbb{T} ; \mathbb{C})=\left\{x: \mathbb{R} \rightarrow \mathbb{C}, 2 \pi-\text { periodic, } x^{\prime}, x^{\prime \prime} \in L^{2}(\mathbb{T} ; \mathbb{C})\right\},
\end{gathered}
$$

where $x^{\prime}=\frac{d x}{d \xi}$ and $x^{\prime \prime}=\frac{d^{2} x}{d \xi^{2}}$.

Further we consider the operator $A_{0}: D\left(A_{0}\right)=H^{2}(\mathbb{T} ; \mathbb{C}) \rightarrow L^{2}(\mathbb{T} ; \mathbb{C})$ so defined

$$
A_{0} x=x^{\prime \prime}, x \in H^{2}(\mathbb{T} ; \mathbb{C})
$$

and we assume that the operator $A_{0}$ is the infinitesimal generator of a strongly continuous cosine family $\left\{C_{0}(t)\right\}_{t \in \mathbb{R}}$, where $C_{0}(t): L^{2}(\mathbb{T} ; \mathbb{C}) \rightarrow L^{2}(\mathbb{T} ; \mathbb{C})$, for every $t \in \mathbb{R}$ (see references in [11]).

Moreover we fix the function $P: I \rightarrow \mathcal{L}\left(H^{1}(\mathbb{T} ; \mathbb{C}) ; L^{2}(\mathbb{T} ; \mathbb{C})\right)$ defined in this way 


$$
P(t) x=b(t) x^{\prime}, t \in I, x \in H^{1}(\mathbb{T} ; \mathbb{C})
$$

where $b: I \rightarrow \mathbb{R}$ is of class $C^{1}$ on $I$.

Now we are in a position to define the family $\{A(t): t \in I\}$ where, for every $t \in I, A(t): H^{2}(\mathbb{T} ; \mathbb{C}) \rightarrow L^{2}(\mathbb{T} ; \mathbb{C})$ is an operator so defined

$$
A(t) \doteq A_{0}+P(t), t \in I .
$$

In Theorem 1.2 of [10], Henríquez has proved that this family generates a fundamental system $\{S(t, s)\}_{(t, s) \in I \times I}$, which is also compact (see [11], $\S 4$ ).

Moreover, let us consider $p: I \rightarrow \mathbb{C}$ a continuous map, $\varphi_{1}, \varphi_{2}: I \rightarrow \mathbb{C}$ such that

$(\varphi) \varphi_{1}, \varphi_{2} \in L^{1}(I ; \mathbb{C})$;

and the map $f: I \times \mathbb{C} \rightarrow \mathbb{C}$ having the following properties:

$(f 1) f(t, x().) \in L^{2}(\mathbb{T} ; \mathbb{C})$, for every $t \in I$ and for every $x \in L^{2}(\mathbb{T} ; \mathbb{C})$;

$(f 2)$ for every $x \in L^{2}(\mathbb{T} ; \mathbb{C})$, the map $t \rightarrow f(t, x()$.$) is weakly measurable;$

(f3) there exists $\alpha \in L_{+}^{1}(I)$ such that, for every $k=1,2$, we have

$$
\|f(t, z)-f(t, w)\|_{\mathbb{C}} \leq \alpha(t)\|z-w\|_{\mathbb{C}} \text {, for a.e. } t \in I \text { and for every } z, w \in \mathbb{C} \text {; }
$$

(f4) $\quad f(., 0) \in L^{1}(I ; \mathbb{C})$.

In what follows we revise functions $w, u: I \times \mathbb{R} \rightarrow \mathbb{C}$ such that $w(t,),. u(t,.) \in$ $L^{2}(\mathbb{T} ; \mathbb{C})$, for every $t \in I$, as two maps $x, v: I \rightarrow L^{2}(\mathbb{T}, \mathbb{C})$ respectively so defined

$$
\begin{aligned}
& x(t)(\xi)=w(t, \xi), t \in I, \quad \xi \in \mathbb{R} \\
& v(t)(\xi)=u(t, \xi), t \in I, \quad \xi \in \mathbb{R} .
\end{aligned}
$$

Now we define the function $\tilde{f}: I \times L^{2}(\mathbb{T} ; \mathbb{C}) \rightarrow L^{2}(\mathbb{T} ; \mathbb{C})$ such that

$$
\tilde{f}(t, x)(\xi)=f(t, x(\xi)), t \in I, x \in L^{2}(\mathbb{T} ; \mathbb{C}), \xi \in \mathbb{R}
$$

and by hypothesis $(f 1)$ we have that $\tilde{f}$ is correctly defined.

Next we consider the multimap $\widetilde{U}: I \rightarrow \mathcal{P}\left(L^{2}(\mathbb{T} ; \mathbb{C})\right)$ so defined

$$
\widetilde{U}(t)=\left[\tilde{\varphi}_{1}(t), \tilde{\varphi}_{2}(t)\right] \doteq\left\{\lambda \tilde{\varphi}_{1}(t)+(1-\lambda) \tilde{\varphi}_{2}(t): \lambda \in[0,1]\right\}, t \in I,
$$

where, for every $t \in I$ and $i=1,2, \tilde{\varphi}_{i}(t): \mathbb{R} \rightarrow \mathbb{C}$ is given by

$$
\tilde{\varphi}_{i}(t)(\xi) \doteq \varphi_{i}(t), \xi \in \mathbb{R} .
$$

Since obviously for every $t \in I, \tilde{\varphi}_{1}(t), \tilde{\varphi}_{2}(t) \in L^{2}(\mathbb{T} ; \mathbb{C})$, the multimap $\widetilde{U}$ is well posed. 
Moreover we define the multimap $F: I \times L^{2}(\mathbb{T}, \mathbb{C}) \rightarrow \mathcal{P}\left(L^{2}(\mathbb{T} ; \mathbb{C})\right)$ in the following way (see $(5.6,(5.7))$ )

$$
F(t, x) \doteq\{\tilde{f}(t, x)+v: v \in \tilde{U}(t)\}, t \in I, x \in L^{2}(\mathbb{T} ; \mathbb{C}) .
$$

Finally we introduce the function $N: I \rightarrow C\left(C\left(I ; L^{2}(\mathbb{T} ; \mathbb{C})\right) ; L^{2}(\mathbb{T} ; \mathbb{C})\right)$ such that, for every $t \in I$ and for every $h \in C\left(I ; L^{2}(\mathbb{T} ; \mathbb{C})\right)$, the map $N(t)(h)$ is so defined

$$
N(t)(h)(\xi)=\left\{\begin{array}{l}
\|h\|_{\infty} \int_{0}^{t} p(s) d s,\|h\|_{\infty} \leq 1 \\
\int_{0}^{t} p(s) d s \quad,\|h\|_{\infty}>1,
\end{array}\right.
$$

for every $\xi \in \mathbb{R}$.

Clearly, for every $t \in I$ and $h \in C\left(I ; L^{2}(\mathbb{T} ; \mathbb{C})\right)$, being the map $N(t)(h)$ constant on $\mathbb{T}$, we have that $N(t)(h) \in L^{2}(\mathbb{T}, \mathbb{C})$ and therefore $N$ is also correctly defined.

So, by recalling $(5.8, \quad(5.2), \quad(5.3), \quad(5.4), \quad(5.11), \quad(5.9))$ and (5.12), problem (5.1) can be rewritten in the abstract form

$$
\left\{\begin{array}{l}
x^{\prime \prime}(t) \in A_{0} x(t)+P(t) x(t)+F(t, N(t)(x))=A(t) x(t)+F(t, N(t)(x)), t \in I \\
x(0)=\hat{x}_{0} \\
x^{\prime}(0)=\hat{x}_{1},
\end{array}\right.
$$

where $\hat{x}_{0}, \hat{x}_{1}: \mathbb{R} \rightarrow \mathbb{C}$ are the functions of $L^{2}(\mathbb{T} ; \mathbb{C})$ respectively defined $\hat{x}_{0}(\xi)=$ $x_{0}, \hat{x}_{1}(\xi)=x_{1}$, for every $\xi \in \mathbb{R}$.

At this point let us show that we can apply the Cauchy version of our Theorem 3.5 (see Remark 3.7) .

By recalling the continuity of the function $p: I \rightarrow \mathbb{C}$, after some standard calculations, we can say that, for every $h \in C\left(I ; L^{2}(\mathbb{T} ; \mathbb{C})\right)$, the map $N().(h)$ is even lipschitzian, so $N$ satisfies $(N 1)$. Moreover the continuity of $p$ implies that $N$ has also property $(N 2)_{s}$.

Now we prove that $(F N)$ holds. Indeed, from the definition of the norm in $L^{2}(\mathbb{T} ; \mathbb{C})$ and by bearing in mind hypotheses $(f 3),(f 4)$ and $(\varphi)$, we can write (see $(5.10,(5.8)$ and $(5.12)))$

$$
\begin{aligned}
\|F(t, N(t)(h))\| \leq & \left(\int_{0}^{2 \pi}\|f(t, N(t)(h)(\xi))\|_{\mathbb{C}}^{2} d \xi\right)^{\frac{1}{2}} \\
& +\left(\int_{0}^{2 \pi}\left\|\varphi_{1}(t)\right\|_{\mathbb{C}}^{2} d \xi\right)^{\frac{1}{2}} \\
& +\left(\int_{0}^{2 \pi}\left\|\varphi_{2}(t)\right\|_{\mathbb{C}}^{2} d \xi\right)^{\frac{1}{2}}
\end{aligned}
$$




$$
\begin{aligned}
\leq & \left(\int_{0}^{2 \pi}\left[\alpha(t) \min \left\{1 ;\|h\|_{\infty}\right\}\left\|\int_{0}^{t} p(s) d s\right\|_{\mathbb{C}}+\|f(t, 0)\|_{\mathbb{C}}\right]^{2} d \xi\right)^{\frac{1}{2}} \\
& +\sqrt{2 \pi}\left(\left\|\varphi_{1}(t)\right\|_{\mathbb{C}}+\left\|\varphi_{2}(t)\right\|_{\mathbb{C}}\right) \\
\leq & \sqrt{2 \pi}\left[\alpha(t) P T+\|f(t, 0)\|_{\mathbb{C}}+\left\|\varphi_{1}(t)\right\|_{\mathbb{C}}+\left\|\varphi_{2}(t)\right\|_{\mathbb{C}}\right] \doteq \hat{\alpha}(t),
\end{aligned}
$$

where the function $\hat{\alpha}: I \rightarrow \mathbb{R}^{+}$belongs to $L_{+}^{1}(I)$, since $\alpha,\|f(., 0)\|_{\mathbb{C}} \in L_{+}^{1}(I)$ and $P=\max _{s \in[0, T]}\|p(s)\|_{\mathbb{C}}$. Therefore we can conclude that $(F N)$ condition is satisfied (with function $\psi: \mathbb{R}^{+} \rightarrow \mathbb{R}^{+}$identically egual to 1 ).

Moreover, having $\widetilde{U}$ compact and convex values, we can say that also $F$ takes compact and convex values.

On the other hand $F$ satisfies hypothesis $(F 1)$, i.e. for every $x \in L^{2}(\mathbb{T} ; \mathbb{C})$, $F(., x)$ has a strongly measurable selection. Indeed, from $(f 2)$ and by the separability of $L^{2}(\mathbb{T} ; \mathbb{C})$, we have that, for every $x \in L^{2}(\mathbb{T} ; \mathbb{C}), \tilde{f}(., x)$ is strongly measurable (see [5], Corollary 3.10.5). Then by the strong measurability of $\varphi_{i}$, we get that $\tilde{\varphi}_{i}$ is also strongly measurable. Further obviously it appears that $\tilde{\varphi}_{i}(t) \in \widetilde{U}(t)$, for every $t \in I$ (see $\left.(5.9)\right)$. So we can conclude that $\tilde{f}(., x)+\tilde{\varphi}_{i}$ is a strongly measurable selection of $F(., x)$ and hence $(F 1)$ holds.

Now we prove that $F$ satisfies hypotheses $(F 2)$ and $(F 3)$. To this end, let us consider a set $V \subset I$ having null measure and such that inequalities in (f3) are true.

Firstly, in order to have $(F 2)$, fixed $t \in I \backslash V$, we define following multimaps

$$
\begin{aligned}
G_{t}: L^{2}(\mathbb{T} ; \mathbb{C}) & \rightarrow \mathcal{P}_{k}\left(L^{2}(\mathbb{T} ; \mathbb{C})\right) \\
x & \rightarrow G_{t}(x)=\{\tilde{f}(t, x)\}
\end{aligned}
$$

and

$$
\begin{aligned}
H_{t}: L^{2}(\mathbb{T} ; \mathbb{C}) & \rightarrow \mathcal{P}_{k}\left(L^{2}(\mathbb{T} ; \mathbb{C})\right) \\
x & \rightarrow H_{t}(x)=\widetilde{U}(t) .
\end{aligned}
$$

Now we show that $\tilde{f}(t,$.$) is continuous for every \bar{x} \in L^{2}(\mathbb{T} ; \mathbb{C})$. Fixed $\left(x_{n}\right)_{n}$ a sequence in $L^{2}(\mathbb{T} ; \mathbb{C})$ such that $x_{n} \rightarrow \bar{x}$ in $L^{2}(\mathbb{T} ; \mathbb{C})$, by applying $(f 3)$ we obtain

$$
\left\|\tilde{f}\left(t, x_{n}\right)-\tilde{f}(t, \bar{x})\right\|_{L^{2}} \leq \alpha(t)\left\|x_{n}-\bar{x}\right\|_{L^{2}}, \text { for every } n \in \mathbb{N}
$$

so $\tilde{f}\left(t, x_{n}\right)$ converges to $\tilde{f}(t, \bar{x})$ in $L^{2}(\mathbb{T} ; \mathbb{C})$. Therefore $\tilde{f}(t,$.$) is continuous in$ $L^{2}(\mathbb{T} ; \mathbb{C})$, so the multimap $G_{t}$ is upper semicontinuous in $L^{2}(\mathbb{T} ; \mathbb{C})$.

Moreover also $H_{t}$ is upper semicontinuous in $L^{2}(\mathbb{T} ; \mathbb{C})$, being a constant multimap. Since $G_{t}$ and $H_{t}$ have also compact values, we are in the hypotheses of Proposition 2.59 of [12], so we can conclude that the multimap $F(t,)=$. $G_{t}+H_{t}$ is u.s.c. in $L^{2}(\mathbb{T} ; \mathbb{C})$. Therefore, for almost every $t \in I, F(t,$.$) is u.s.c.$ in $L^{2}(\mathbb{T} ; \mathbb{C})$, i.e. $(F 2)$ holds. 
Secondly, we show $(F 3)$.

Fixed $t \in I \backslash V$, we consider the multimap

$$
\begin{aligned}
B_{t}: L^{2}(\mathbb{T} ; \mathbb{C}) \times L^{2}(\mathbb{T} ; \mathbb{C}) & \rightarrow \mathcal{P}_{k c}\left(L^{2}(\mathbb{T} ; \mathbb{C})\right) \\
(x, y) & \rightarrow B_{t}(x, y)=\{\tilde{f}(t, y)\}+\widetilde{U}(t) .
\end{aligned}
$$

First of all, by using the Hausdorff metric $\mathcal{H}$ and by recalling ( f3) and (5.8), for every $x, y_{1}, y_{2} \in L^{2}(\mathbb{T} ; \mathbb{C})$, it is easy to check that

$$
\mathcal{H}\left(B_{t}\left(x, y_{1}\right), B_{t}\left(x, y_{2}\right)\right) \leq\left\|\tilde{f}\left(t, y_{1}\right)-\tilde{f}\left(t, y_{2}\right)\right\|_{L^{2}} \leq \alpha(t)\left\|y_{1}-y_{2}\right\|_{L^{2}} .
$$

Hence the multimap $B_{t}(x,$.$) is \alpha(t)$-Lipschitz, for every $x \in L^{2}(\mathbb{T} ; \mathbb{C})$.

Further, fixed a bounded set $\Omega \subset L^{2}(\mathbb{T} ; \mathbb{C})$ and $y \in L^{2}(\mathbb{T} ; \mathbb{C})$, we have that $B_{t}(\Omega \times\{y\})$ is compact. So, in virtue of Proposition 2.2.2 of [13] the multimap

$$
\begin{aligned}
A_{t}: L^{2}(\mathbb{T} ; \mathbb{C}) & \rightarrow \mathcal{P}_{k}\left(L^{2}(\mathbb{T} ; \mathbb{C})\right) \\
x & \rightarrow A_{t}(x)=B_{t}(x, x)=\{\tilde{f}(t, x)\}+\widetilde{U}(t)
\end{aligned}
$$

is $(\alpha(t), \eta, \eta)$-bounded in $\mathcal{P}_{b}\left(L^{2}(\mathbb{T} ; \mathbb{C})\right)\left(\right.$ where $\eta$ is the MNC in $\left.L^{2}(\mathbb{T} ; \mathbb{C})\right)$, i.e.

$$
\eta\left(A_{t}(\Omega)\right) \leq \alpha(t) \eta(\Omega), \forall \Omega \in \mathcal{P}_{b}\left(L^{2}(\mathbb{T} ; \mathbb{C})\right) .
$$

Hence by $(5.11,(5.14)$ and $(5.15))$ we can say that

$$
\eta(F(t, \Omega))=\eta(\tilde{f}(t, \Omega)+\widetilde{U}(t))=\eta\left(A_{t}(\Omega)\right) \leq \alpha(t) \eta(\Omega), \forall \Omega \in \mathcal{P}_{b}\left(L^{2}(\mathbb{T} ; \mathbb{C})\right) .
$$

So we can conclude that $F$ satisfies hypothesis $(F 3)$ (with $m(t) \doteq \alpha(t), \forall t \in I$ ).

By means of the arguments above presented, we are in a position to apply the Cauchy version of our Theorem 3.5. Then we can deduce that there exists a continuous function $\hat{x}: I \rightarrow L^{2}(\mathbb{T} ; \mathbb{C})$ that is a mild solution for $(5.13)$ problem, i.e.

$$
\hat{x}(t)=C(t, 0) x_{0}+S(t, 0) x_{1}+\int_{0}^{t} S(t, s) \hat{q}(s) d s, t \in I,
$$

where $\hat{q} \in S_{F, N}^{1}(\hat{x})=\left\{f \in L^{1}\left(I ; L^{2}(\mathbb{T}, \mathbb{C})\right): f(t) \in F(t, N(t)(\hat{x}))\right.$ a.e. $\left.t \in I\right\}$.

Therefore, since $\hat{q}(t) \in F(t, N(t)(\hat{x}))$, a.e. $t \in I$, there exists $v_{\hat{x}}(t) \in \widetilde{U}(t)$ such that

$$
v_{\hat{x}}(t)=\hat{q}(t)-\tilde{f}(t, N(t)(\hat{x})) \text {, a.e } t \in I,
$$

which is strongly measurable, being $\hat{q}$ and $\tilde{f}(., N().(\hat{x}))$ strongly measurable (see [13], Theorem 1.3.5). 
At this point, by considering functions $w: I \times \mathbb{R} \rightarrow \mathbb{C}$ and $u: I \times \mathbb{R} \rightarrow \mathbb{C}$ respectively so defined

$$
\begin{aligned}
& w(t, \xi)=\hat{x}(t)(\xi), t \in I, \quad \xi \in \mathbb{R} \\
& u(t, \xi)=v_{\hat{x}}(t)(\xi), t \in I, \quad \xi \in \mathbb{R}
\end{aligned}
$$

we can conclude that $\{w, u\}$ is an admissible mild-pair for (5.1) problem.

Finally we are able to enunciate the following result.

Theorem 5.1. In the framework above described, there exists an admissible mild-pair for (5.1) problem, i.e. functions $w$ and $u$ satisfying following properties:

$(w 1)$ for every $t \in I, w(t,$.$) is 2 \pi$-periodic and 2 -integrable on $[0,2 \pi]$;

$(w 2)$ for every $\xi \in \mathbb{R}, w(., \xi)$ is continuous on $I$;

$(w 3) w(0, \xi)=x_{0}$, for every $\xi \in \mathbb{R}$;

$(w 4)$ for every $\xi \in \mathbb{R}$ such that $w(., \xi)$ is derivable in 0 then $\frac{\partial w}{\partial t}(0, \xi)=x_{1}$;

(u1) for every $\xi \in \mathbb{R}, u(., \xi)$ is strongly measurable and such that $u(t, \xi) \in\left[\varphi_{1}(t), \varphi_{2}(t)\right]$, a.e. $t \in I$ and for every $\xi \in \mathbb{R}$.

Moreover, they are such that

$$
w(t, \xi)=C(t, 0) x_{0}+S(t, 0) x_{1}+\int_{0}^{t} S(t, s) q(s, \xi) d s, t \in I, \quad \xi \in \mathbb{R},
$$

where $q: I \times \mathbb{R} \rightarrow \mathbb{C}$ is so defined

$$
q(t, \xi)=f\left(t, \min \left\{1 ; \max _{s \in I}\|w(s, .)\|_{L^{2}}\right\} \int_{0}^{t} p(s) d s\right)+u(t, \xi), t \in I, \xi \in \mathbb{R} .
$$

\section{Acknowledgements}

The research is carried out within the national group GNAMPA of INdAM. The first author is partially supported by the Department of Mathematics and Computer Science of the University of Perugia (Italy) and by the projects "Metodi della Teoria dell'Approssimazione, Analisi Reale, Analisi Nonlineare e loro applicazioni" and "Integrazione, Approssimazione, Analisi Nonlineare e loro Applicazioni", funded by the 2018 and 2019 basic research fund of the University of Perugia, and by a 2020 GNAMPA-INDAM Project "Processi evolutivi con memoria descrivibili tramite equazioni integro-differenziali".

Funding Open access funding provided by Università degli Studi di Perugia within the CRUI-CARE Agreement. 
Open Access. This article is licensed under a Creative Commons Attribution 4.0 International License, which permits use, sharing, adaptation, distribution and reproduction in any medium or format, as long as you give appropriate credit to the original author(s) and the source, provide a link to the Creative Commons licence, and indicate if changes were made. The images or other third party material in this article are included in the article's Creative Commons licence, unless indicated otherwise in a credit line to the material. If material is not included in the article's Creative Commons licence and your intended use is not permitted by statutory regulation or exceeds the permitted use, you will need to obtain permission directly from the copyright holder. To view a copy of this licence, visit http://creativecommons. org/licenses/by/4.0/.

\section{Appendix}

In this paper $X$ is a Banach space with the norm $\|\cdot\|_{X}$ and, if $\mathcal{P}(X)$ is the family of all non-empty subsets of $X$, we will use the following notations:

$$
\begin{aligned}
& \mathcal{P}_{b}(X)=\{H \in \mathcal{P}(X): H\text { bounded }\}, \\
& \mathcal{P}_{c}(X)=\{H \in \mathcal{P}(X): H \quad \text { convex }\}, \\
& \mathcal{P}_{f}(X)=\{H \in \mathcal{P}(X): H \quad \text { closed }\}, \\
& \mathcal{P}_{k}(X)=\{H \in \mathcal{P}(X): H \quad \text { compact }\}, \\
& \mathcal{P}_{f c}(X)=\mathcal{P}_{f}(X) \cap \mathcal{P}_{c}(X), \\
& \mathcal{P}_{k c}(X)=\mathcal{P}_{k}(X) \cap \mathcal{P}_{c}(X), \\
& \\
&{ } \\
& \mathcal{P}_{b f c}(X)= \mathcal{P}_{b}(X) \cap \mathcal{P}_{f}(X) \cap \mathcal{P}_{c}(X) .
\end{aligned}
$$

Further, let $I=[0, T]$ be an interval of the real line endowed with the usual Lebesgue measure $\mu$.

A function $f: I \rightarrow X$ is said to be strongly measurable if there exists a sequence $\left(f_{n}\right)_{n}, f_{n}: I \rightarrow X$, of simple functions which converges to $f$ almost everywhere in $I$ (see [5], Definition 3.10.1 (a)). A function $f: I \rightarrow X$ is called weakly measurable if for every $x^{*} \in X^{*}$, the $\mathbb{R}$-valued function $t \rightarrow<x^{*}, f(t)>$ is measurable (where $X^{*}$ is the dual topological space of $X$ ) (see [5], Definition $3.10 .1(\mathrm{~b}))$.

Moreover, we denote by $C(I ; X)$, the space consisting of all continuous functions from $I$ to $X$ provided with the norm $\|.\|_{\infty}$ of the uniform convergence, by $L^{1}(I ; X)$ the space of all $X$-valued Bochner-integrable functions on $I$ with the norm $\|u\|_{1}=\int_{0}^{T}\|u(t)\|_{X} d t$ and by $L_{+}^{1}(I)=\left\{f \in L^{1}(I ; \mathbb{R}): f(t) \geq 0\right.$, for a.e. $t \in I\}$.

A countable set $\left\{f_{n}\right\}_{n} \subset L^{1}(I ; X)$ is said to be semicompact if (i) $\left\{f_{n}\right\}_{n}$ is integrably bounded, i.e. there exists $\omega \in L_{+}^{1}(I)$ such that $\left\|f_{n}(t)\right\| \leq \omega(t)$, for a.e. $t \in I$ and for every $n \in \mathbb{N}$; (ii) the set $\left\{f_{n}(t)\right\}_{n}$ is relatively compact in $X$, for a.e. $t \in I$. 
A multimap $F: I \rightarrow \mathcal{P}(X)$ is measurable if the set

$$
F^{-}(A)=\{t \in I: F(t) \cap A \neq \emptyset\}
$$

is measurable, for each open subset $A$ of $X$.

A multimap $F: I \rightarrow \mathcal{P}(X)$ is said to be graph measurable if $G r F=$ $\{(t, x) \in I \times X: x \in F(t)\} \in \Sigma \times \mathcal{B}(X)$, where $\Sigma$ is the $\sigma$-algebra on $I$ and $\mathcal{B}(X)$ is the Borel $\sigma$-algebra on $X$.

A multimap $F: I \rightarrow \mathcal{P}_{b}(X)$ is said to have a strongly measurable selection if there exists a strongly measurable function $f: I \rightarrow X$ such that $f(t) \in$ $F(t)$, for almost everywhere $t \in I$.

Furthermore a multimap $F: X \rightarrow \mathcal{P}(X)$ is called totally bounded if $F$ maps bounded subsets of $X$ into relatively compact sets of $X$ and $F$ is called locally compact if, for every $x \in X$, there exists a neighbourhood $U$ of the point $x$ such that $F(U)$ is relatively compact in $X$.

Next the multimap $F$ is called upper semicontinuous (u.s.c.) in $\bar{x} \in X$ if, for every open set $A$ of $X$ such that $F(\bar{x}) \subset A$, there exists a neighborhood $U(\bar{x}): F(x) \subset A$, for every $x \in U(\bar{x})$ and $F: X \rightarrow \mathcal{P}(X)$ is called lower semicontinuous (l.s.c.) in $\bar{x} \in X$ if, for every open set $A$ of $X$ such that $A \cap F(\bar{x}) \neq \emptyset$, there exists a neighborhood $U(\bar{x}): F(x) \cap A \neq \emptyset$, for every $x \in U(\bar{x})$.

Finally a multimap $G: I \times X \rightarrow \mathcal{P}(X)$ is said Scorza-Dragoni lower semicontinuous $((S . D)-$. l.s.c. $)$ if, for every $\epsilon>0$, there exists a closed set $T_{\epsilon} \subseteq I: \mu\left(I \backslash T_{\epsilon}\right)<\epsilon$ such that $\left.G\right|_{T_{\epsilon} \times X}$ is lower semicontinuous.

Now we enunciate the following well-known fixed point theorem proved by Martelli in [15].

Theorem 6.1. Let $X$ be a separable Banach space and let $F: X \rightarrow \mathcal{P}_{b f c}(X)$ be a multimap such that

(i) $F$ is totally bounded and upper semicontinuous;

(ii) The set $\Omega=\{x \in X: \exists \lambda>1$ such that $\lambda x \in F(x)\}$ is bounded.

Then $F$ has at least a fixed point.

Next, let us denote by $O_{n}$ the zero-element of $\mathbb{R}^{n}$ and by $\preccurlyeq$ the partial ordering given by the standard positive cone $\mathbb{R}_{0,+}^{n} \doteq\left(\mathbb{R}_{0}^{+}\right)^{n}$, i.e. $x \preccurlyeq y$ if and only if $y-x \in \mathbb{R}_{0,+}^{n}$; clearly, $x \prec y$ means that $x \preccurlyeq y$ and $x \neq y$.

Moreover we present the concept of measure of noncompactness in $X$ (see [13], Definition 2.1.1).

Definition 6.2. A function $\eta: \mathcal{P}_{b}(X) \rightarrow \mathbb{R}_{0,+}^{n}$ is said to be a measure of noncompactness (MNC, for short) in the Banach space $X$ if, for every $\Omega \in \mathcal{P}_{b}(X)$, the following properties are satisfied:

$\left(\eta_{1}\right) \eta(\Omega)=0$ if and only if $\bar{\Omega}$ is compact;

$\left(\eta_{2}\right) \eta(\overline{c o}(\Omega))=\eta(\Omega)$, for all $\Omega \in \mathcal{P}_{b}(X)$. 
Further, a MNC $\eta: \mathcal{P}_{b}(X) \rightarrow \mathbb{R}_{0,+}^{n}$ is said to be monotone if $\Omega_{1}, \Omega_{2} \in \mathcal{P}_{b}(X): \Omega_{1} \subset \Omega_{2}$ implies $\eta\left(\Omega_{1}\right) \preccurlyeq \eta\left(\Omega_{2}\right)$.

We recall that the Hausdorff measure of noncompactness has the mentioned property.

\section{References}

[1] Aizicovici, S., Staicu, V.: Multivalued evolution equations with nonlocal initial conditions in Banach spaces. NoDEA Nonlinear Differ. Equ. Appl. 14, 361-376 (2007)

[2] Byszewski, L.: Theorems about the existence and uniqueness of solutions of a semilinear evolution nonlocal Cauchy problem. J. Math. Anal. Appl. 162, 494505 (1991)

[3] Cardinali, T., Gentili, S.: An existence theorem for a non-autonomous second order nonlocal multivalued problem. Stud. Univ. Babes-Bolyai Math. 62, 101117 (2017)

[4] Cascales, C., Kadets, V., Rodriguez, J.: Measurability and selections of multifunctions in Banach spaces. J. Convex Anal. 17, 229-240 (2010)

[5] Denkowski, Z., Migorski, S., Papageorgiou, N.S.: An Introduction to Nonlinear Analysis. Kluwer Academic Publishers, New York (2003)

[6] Fattorini, H.O.: Second Order Linear Differential Equations in Banach Spaces. North-Holland Publishing Co., Amsterdam (1985)

[7] Fokas, A.S., Keller, J.B., Clarkson, B.D.: A mathematical model of granulocytopoiesis and chronic myelogenous leukemia. Cancer 51, 2084-2091 (1991)

[8] García-Falset, J.: Existence results and asymptotic behaviour for nonlocal abstact Cauchy problems. J. Math. Anal. Appl. 338, 639-652 (2008)

[9] García-Falset, J., Reich, S.: Integral solutions to a class of nonlocal evolution equations. Commun. Contemp. Math. 12, 1032-1054 (2010)

[10] Henríquez, H.R.: Existence of solutions of non-autonomous second order functional differential equations with infinite delay. Nonlinear Anal. 74, 3333-3352 (2011)

[11] Henríquez, H.R., Poblete, V., Pozo, J.C.: Mild solutions of non-autonomous second order problems with nonlocal initial conditions. J. Math. Anal. Appl. 412, 1064-1083 (2014)

[12] Hu, S., Papageorgiou, N.S.: Handbook of Multivalued Analysis. Theory, vol. I. Kluwer Academic Publishers, Dordrecht (1997)

[13] Kamenskii, M., Obukhovskii, V., Zecca, P.: Condensing Multivalued Maps and Semilinear Differential Inclusions in Banach Spaces. De Gruyter Series in Nonlinear Analysis and Applications, vol. 7. Walter de Gruyter, Berlin (2001)

[14] Kozak, M.: A fundamental solution of a second-order differential equation in a Banach space. Univ. Iagel. Acta Math. 32, 275-289 (1995)

[15] Martelli, M.: A Rothe's type theorem for noncompact acyclic-valued map. Boll. Unione Matemat. Ital. 11, 70-76 (1975) 
[16] McKibben, M.: Discovering Evolution Equations with Applications. CRC Applied Mathematics Nonlinear Science Series, Deterministic Models, vol. I. Chapman \& Hall, London (2011)

[17] Moore, H., Li, N.K.: A mathematical model of chronic myelogenous leukemia (CML) and T cell interaction. J. Theor. Biol. 227, 513-523 (2004)

[18] Paicu, A., Vrabie, I.I.: A class of nonlinear evolution equations subjected to nonlocal initial conditions. Nonlinear Anal. 72, 4091-4100 (2010)

[19] Tolstonogov, A.: Differential Inclusions in a Banach Space. Kluwer Academic Publishers, Dordrecht (2000)

[20] Vincent, P.C., Cronkite, E.P., Greenberg, M.L., Kirsten, C., Schiffer, L.M., Stryckmans, P.A.: Leukocyte kinetics in chronic myeloid leukemia. I. DNA synthesis time in blood and marrow myelocytes. Blood 33, 843-850 (1969)

Tiziana Cardinali and Eleonora De Angelis

Department of Mathematics and Computer Sciences

University of Perugia

via L. Vanvitelli 1

06123 Perugia

Italy

e-mail: tiziana.cardinali@unipg.it;

ele2994@tiscali.it

Received: December 26, 2019.

Accepted: October 16, 2020.

Publisher's Note Springer Nature remains neutral with regard to jurisdictional claims in published maps and institutional affiliations. 